Proceedings of the 2011 Winter Simulation Conference

S. Jain, R. R. Creasey, J. Himmelspach, K. P. White, and M. Fu, eds.

\title{
RARE EVENT SIMULATION TECHNIQUES
}

\author{
Jose Blanchet \\ Columbia University \\ 500 W 120th Street \\ New York, NY 10027, USA
}

\author{
Henry Lam \\ Boston University \\ 111 Cummington Street \\ Boston, MA 02215, USA
}

\begin{abstract}
We discuss rare event simulation techniques based on state-dependent importance sampling. Classical examples and counter-examples are shown to illustrate the reach and limitations of the state-independent approach. State-dependent techniques are helpful to deal with these limitations. These techniques can be applied to both light and heavy tailed systems and often are based on subsolutions to an associated Isaacs equation and on Lyapunov bounds.
\end{abstract}

\section{INTRODUCTION}

We study recent techniques that have been developed for the design of provably efficient rare event simulation estimators. We concentrate mostly on importance sampling techniques. Our discussion here is based on the survey paper Blanchet and Lam (2011).

We mention that besides importance sampling, another powerful set of tools for variance reduction in rare event problems are based on splitting. Interested readers can consult L'Ecuyer et al. (2009) and Chapter VI Section 9 of Asmussen and Glynn (2007) for basic notions on splitting.

The types of techniques that we consider in this paper focus on the interplay between large deviations results and their applications to provably efficient importance sampling estimators. Early references in this spirit include Siegmund (1976) and Sadowsky (1991). Applications of these techniques in settings such as insurance and queueing include Asmussen (1985), Collamore (2002), Asmussen and Rubinstein (1995), and Parekh and Walrand (1989). We refer the reader to Juneja and Shahabuddin (2006), Bucklew (1990) and Chapter VI of Asmussen and Glynn (2007) for a wider scope on importance sampling for rare event simulation.

A series of examples and counter-examples (Glasserman and Kou 1995, Glasserman and Wang 1997) related to the use of large deviations principles in the design of efficient importance sampling estimators for light-tailed systems has motivated research on state-dependent techniques. In addition, as explained in Bassamboo et al. (2006), efficient importance sampling estimators in the setting of heavy tails often must be state-dependent (see also Asmussen et al. 2000 for more on the challenges that arise in the context of efficient rare-event simulation for heavy-tailed systems).

In order to systematically address the construction of provably efficient state-dependent importance sampling estimators for light-tailed systems P. Dupuis, H. Wang and their students have developed a method based on control theory and the use of subsolutions of an associated Isaacs equation for constructing and testing importance sampling estimators (Dupuis and Wang 2004; Dupuis and Wang 2007; Dupuis et al. 2007; Dupuis et al. 2007). A related approach, based on the construction of Lyapunov inequalities has been also developed (Blanchet and Glynn 2008; Blanchet and Liu 2008; Blanchet et al. 2007) for the construction and analysis of state-dependent importance sampling estimators for heavy-tailed systems.

In this paper we review the development of state-dependent importance sampling estimators and illustrate their application. We start by discussing standard notions of statistical efficiency that are used in order to rigorously test the optimality of a simulation estimator. This will be done in Section 2. The introduction of 


\section{Blanchet and Lam}

these notions of efficiency leads to the question of how to construct importance sampling estimators that can be rigorously shown to be efficient. The zero-variance change-of-measure, while virtually impossible to implement in most situations of interest, provides a good guidance in order to construct such estimators.

The zero-variance change-of-measure turns out to be the conditional distribution of the underlying process given the rare event of interest. So, it is natural to leverage-off the available asymptotic theory for rare events in order to understand such a conditional distribution. This is basically the starting point in the design of efficient importance sampling estimators for rare events. The idea is to take advantage of the available large deviations theory, which provides insight into the occurrence of rare events. In the light-tailed case, the theory of large deviations is comprehensively documented in Dembo and Zeitouni (1998). References in connection to large deviations for heavy-tailed problems (mainly related to random walk settings) can be found in Embrechts et al. (1997) and Chapter IX of Asmussen (2000).

The successful application of large deviations theory in the design of efficient importance sampling estimators is not straightforward and this feature turned out to be the norm rather than the exception, especially in applications such as the analysis of queueing networks (see Glasserman and Kou 1995). To avoid such poor variance behavior, Dupuis and Wang formulate the design of state-dependent importance sampling estimators in terms of a stochastic control problem in which the value function is the second moment of the estimator. Taking an asymptotic limit, as we shall see in Section 3, the stochastic control problem becomes a deterministic control problem. The application of Dupuis and Wang's method is simplified thanks to the fact that only a subsolution to the value function is needed for the design of an efficient sampling scheme. A trivial supersolution is given, thanks to Jensen's inequality, by twice the underlying large deviations rate function. The whole point of Dupuis and Wang's approach is to construct a subsolution that matches the trivial supersolution at one point, namely, the initial condition of the system under consideration. In many problems of interest it turns out that such a subsolution is piecewise affine and therefore relatively easy to manipulate.

We will then move to the analysis of heavy-tailed systems, which will be introduced in our last section, namely Section 4. These types of systems arise in the analysis of finance and insurance models. There are fundamental qualitative differences between light and heavy tailed systems when it comes to the analysis of rare events. This is why it is incorrect to use a model with light-tailed components to study large deviations properties of systems that are built from heavy-tailed building blocks. Light-tailed large deviations occur gradually, while heavy-tailed large deviations occur suddenly and this feature manifests itself in the design of efficient importance sampling estimators for heavy-tailed systems.

We emphasize that this paper aims to introduce readers the key concepts and ideas in rare-event problems. Throughout the exposition we will drop many of the mathematical details and highlight only the central arguments, pointing readers to other references. Finally, we briefly mention the existence of some empirical approaches aimed at selecting good importance sampling distributions. We do not discuss these methods in detail but rather refer the reader to some related references including: adaptive Monte Carlo (Kollman, Baggerly, Cox, and Picard 1999) and the cross-entropy method (Rubinstein and Kroese 2004). In this vein, we mention the recent work of Chan and Lai (2010) who considers an interesting importance sampling approach for Markov-modulated problems that avoids the need for computing the associated eigenfunctions.

\section{NOTIONS ON EFFICIENCY AND IMPORTANCE SAMPLING}

\subsection{Notions of Efficiency}

In order to design algorithms that achieve high accuracy in relative terms and that perform well in rare event settings it is useful to embed the question of interest in a suitable asymptotic regime, often motivated by an applied standpoint. For example, in the setting of bankruptcy of an insurance company, it is often the case that the capital or reserve of the company is large relative to individual claim sizes, and this is basically why one might expect the probability of bankruptcy, $P(A)$, to be small. So, one might introduce 


\section{Blanchet and Lam}

a parameter, say $n$, for instance the initial capital in the insurance example, and consider $\alpha_{n}=P\left(A_{n}\right)$ as a function of the parameter $n$ which is often referred to as the "rarity parameter". From now on we will assume that underlying the rare event there is a rarity parameter $n$ such that $\alpha_{n} \longrightarrow 0$ as $n \longrightarrow \infty$.

The idea is then to design a simulation estimator whose error is controlled in relative terms as $n \nearrow \infty$ compared to naive (or crude) Monte Carlo, which provides an obvious alternative and, therefore, a general benchmark. Note that naive Monte Carlo involves simulating $N$ (independent and identically distributed) iid Bernoullies, $I_{n}(1), \ldots, I_{n}(N)$, with success parameter $\alpha_{n}$, thereby producing an estimate of the form $\widehat{\alpha}_{n}^{c}(N)=\sum_{j=1}^{N} I_{n}(j) / N$. The super-index " $c$ " that appears in $\widehat{\alpha}_{n}^{c}(N)$ corresponds to crude Monte Carlo. Since $\widehat{\alpha}_{n}^{c}(N)$ is an unbiased estimator of $\alpha_{n}$, its mean squared error equals $\operatorname{Var}\left(\widehat{\alpha}_{n}^{c}(N)\right)^{1 / 2}=$ $\alpha_{n}^{1 / 2}\left(1-\alpha_{n}\right)^{1 / 2} / N^{1 / 2}$. In turn, we obtain a relative mean squared error equal to the coefficient of variation of $\widehat{\alpha}_{n}^{c}(N)$, namely, $C V\left(\widehat{\alpha}_{n}^{c}(N)\right)=\left(1-\alpha_{n}\right)^{1 / 2} /\left(\alpha_{n}^{1 / 2} N^{1 / 2}\right)$. In order to control the relative error of $\widehat{\alpha}_{n}^{c}(N)$ one needs $N=\Omega\left(1 / \alpha_{n}\right)$ Bernoullies.

The problem with the estimator $\widehat{\alpha}_{n}^{c}(N)$ is that each of the underlying replications, the $I_{n}(j)$ 's, have a very big variance in relative terms. The number of replications $N$ must grow at the same rate as $C V\left(I_{n}(j)\right)=\left(1-\alpha_{n}\right)^{1 / 2} / \alpha_{n}^{1 / 2}$. The same phenomenon occurs in any estimator obtained out of averaging iid replications, as in the definition of $\widehat{\alpha}_{n}^{c}(N)$. The objective is then to design an estimator $R_{n}$ with a controlled mean squared error. We concentrate only on unbiased estimators, so controlling the behavior of the mean squared error boils down to controlling the behavior of the second moment. The overall estimate is then obtained by averaging $N$ iid copies of $R_{n}$. Motivated by these considerations we have the following definitions.

Definition 1 An estimator $R_{n}$ is said to be strongly efficient if $E R_{n}^{2}=O\left(\alpha_{n}^{2}\right)$ as $n \nearrow \infty$.

Definition $2 R_{n}$ is said to be weakly efficient or asymptotically optimal if for each $\varepsilon>0$ we have that $E R_{n}^{2}=O\left(\alpha_{n}^{2-\varepsilon}\right)$ as $n \nearrow \infty$.

In most cases the analysis of importance sampling estimators (especially in light-tailed cases) concludes only weak efficiency of the estimators.

In order to see the gain in efficiency, suppose that an estimator $R_{n}$ is strongly efficient and consider $\widetilde{\alpha}_{n}(N)=\sum_{i=1}^{N} R_{n}(i) / N$ where the $R_{n}(i)$ 's are independent replications of $R_{n}$. It follows from Chebyshev's inequality that at most $N=O\left(\varepsilon^{-2} \delta^{-1}\right.$ ) (uniformly in $n$ ) replications are required to conclude that $\widetilde{\alpha}_{n}(N)$ is $\varepsilon$-close to $\alpha_{n}$ in relative terms with at least $1-\delta$ confidence.

Besides variance control, it is worth pointing out the other measures of good importance sampling estimators, and we refer interested readers to L'Ecuyer et al. (2010) for these considerations (including work-normalized measures to account for cost-per replication).

\subsection{Basic Properties of Importance Sampling Estimators}

Importance sampling is a variance reduction technique that is often applied to design an estimator, $R_{n}$, with efficiency characteristics such as those described by Definitions 1 and 2. We shall review basic properties of importance sampling next; for a more detailed discussion see, for example, Rubinstein and Kroese (2009) and Asmussen and Glynn (2007). The basic idea is to introduce a probability measure $\widetilde{P}(\cdot)$ such that the likelihood ratio or Radon-Nikodym derivative between the nominal (original) probability measure, $P(\cdot$ ), and $\widetilde{P}(\cdot)$ is well defined on the event $A_{n}$ of interest. Then we can simply let

$$
R_{n}=\frac{d P}{d \widetilde{P}}(\omega) I\left(\omega \in A_{n}\right)
$$

as usual, we use $\omega$ to denote the underlying random outcome which is simulated according to the probability measure $\widetilde{P}(\cdot)$. We use $\widetilde{E}(\cdot)$ to denote the expectation operator associated to the probability measure $\widetilde{P}(\cdot)$. 


\section{Blanchet and Lam}

Note that

$$
\widetilde{E} R_{n}=\int_{A_{n}} \frac{d P}{d \widetilde{P}}(\omega) d \widetilde{P}(\omega)=P\left(A_{n}\right)=\alpha_{n},
$$

so $R_{n}$ is an unbiased estimator, and

$$
\widetilde{E}\left(R_{n}^{2}\right)=\int_{A_{n}}\left(\frac{d P}{d \widetilde{P}}(\omega)\right)^{2} d \widetilde{P}(\omega)=\int_{A_{n}} \frac{d P}{d \widetilde{P}}(\omega) d P(\omega)=E\left(R_{n}\right) .
$$

In principle one can design an importance sampling estimator with zero variance. Indeed, if we let $\widetilde{P}(\cdot)=P_{n}^{*}(\cdot):=P\left(\cdot \mid A_{n}\right)$, then

$$
R_{n}^{*}:=\frac{d P}{d P_{n}^{*}}(\omega) I\left(\omega \in A_{n}\right)=P\left(A_{n}\right),
$$

which is clearly an exact estimate of $\alpha_{n}=P\left(A_{n}\right)$. Of course, assuming that one has access to $P^{*}(\cdot)$ defeats the point of using simulation to estimate $P\left(A_{n}\right)$. However, the underlying lesson behind the characterization of the zero-variance change-of-measure as the conditional distribution given the rare event of interest is that one can use asymptotic theory to describe $P_{n}^{*}(\cdot)$ as $n \nearrow \infty$.

\section{STATE-DEPENDENT TECHNIQUES FOR LIGHT-TAILED SYSTEMS}

\subsection{Solutions to a Deterministic Control Problem and Efficient Importance Sampling}

As we indicated in the Introduction, Dupuis and Wang introduced a technique to guarantee efficiency (in the sense of Definition 2) of importance sampling estimators based on control theoretic considerations.

We will formally characterize the solution to a deterministic control problem proposed by Dupuis and Wang (2004). In this section we assume that $\left(X_{k}: k \geq 1\right)$ is a sequence of iid rv's taking values in $R^{d}$ and such that $\psi(\theta)=\log E \exp \left(\left\langle X_{k}, \theta\right\rangle\right)$ is finite everywhere, where $\langle\cdot, \cdot\rangle$ is defined as the inner product under Euclidean norm. We then let $S_{k}=X_{1}+\ldots+X_{k}$ and consider, for some $y \in R^{d}$,

$$
Y_{n}(t)=y+S_{\lfloor n t\rfloor} / n .
$$

Let $B$ be a closed set with non-empty interior and define $\tau_{B}(n)=\inf \left\{t>0: Y_{n}(t) \in B\right\}$. We assume that $B$ is attainable in the sense that $P\left(\tau_{B}(n)<\infty \mid Y_{n}(0)=y\right)>0$ for each $y \in R^{d}$.

Consider two disjoint sets $B$ and $C$. We are interested in computing

$$
\alpha_{n}(y)=P_{y}\left(\tau_{B}(n)<\tau_{C}(n), \tau_{B}(n)<\infty\right):=P\left(\tau_{B}(n)<\tau_{C}(n), \tau_{B}(n)<\infty \mid Y_{n}(0)=y\right) .
$$

We assume that $B$ and $C$ are regular in the sense that $\lim _{n \rightarrow \infty} n^{-1} \log \alpha_{n}(y)=-I_{B, C}(y)$, where $I_{B, C}(y)$ is the associated large deviations rate function (which we do not need to specify but can be consulted in Dembo and Zeitouni 1998, p. 176).

We are interested in choosing a suitable importance sampling estimator with optimal performance in the sense of minimizing its second moment. The class of policies that we consider are based on exponential tilting. In particular, the increment $X_{k}$ is ultimately simulated according to the distribution

$$
P_{\theta_{k}}\left(X_{k} \in d x\right)=\exp \left(\left\langle\theta_{k}, X_{k}\right\rangle-\psi\left(\theta_{k}\right)\right) P\left(X_{k} \in d x\right) .
$$

If $X_{k}$ has a density with respect to the Lebesgue measure, say $f(\cdot)$, then $P\left(X_{k} \in d x\right)=f(x) d x$. The notation adopted in (3) is simply a mechanism that allows us to consider general distributions (including discrete or mixtures of discrete and continuous distributions). We will use $E_{\theta_{k}}(\cdot)$ to denote the expectation operator associated to $P_{\theta_{k}}(\cdot)$. The parameter $\theta_{k}$ might depend on $\left(X_{1}, \ldots, X_{k-1}\right)$ but it is not allowed to depend on future observations. We say that $\theta_{k}$ is non-anticipating. As examples of exponentially tilted 


\section{Blanchet and Lam}

distributions consider the following: If $X_{k}$ is a one dimensional standard Gaussian under $P(\cdot)$, then, under $P_{\theta_{k}}(\cdot), X_{k}$ is normal with mean $\theta_{k}$ and unit variance; on the other hand, if under $X_{k}$ is exponentially distributed with unit mean under $P(\cdot)$, then under $P_{\theta_{k}}(\cdot)$ (for $\theta_{k}<1$ ), $X_{k}$ is exponentially distributed with mean $1 /\left(1-\theta_{k}\right)$.

One reason that motivates the use of exponentially tilted distributions is that one can sometimes show that the distribution of $\left(X_{1}, \ldots, X_{k}\right)$ given a large deviations event of interest converges to a suitable optimal exponential tilting as $n \rightarrow \infty$ (see, for instance, Asmussen and Glynn 2007, Section VI.5).

Using exponential tilting the estimator ultimately takes the form

$$
R_{n}=\exp \left(-\sum_{k=1}^{n \tau_{B}(n)}\left\langle\theta_{k}, X_{k}\right\rangle+\sum_{k=1}^{n \tau_{B}(n)} \psi\left(\theta_{k}\right)\right) I\left(\tau_{B}(n)<\tau_{C}(n), \tau_{B}(n)<\infty\right) .
$$

It follows immediately that the HJB (Hamilton-Jacobi-Bellman) equation corresponding to finding the optimal policy (i.e., the optimal sequence of $\theta_{k}$ 's) to minimize the second moment of $R_{n}$ takes the form

$$
\begin{aligned}
V_{n}(y) & =\inf _{\theta} E_{\theta}\left[\exp (-2\langle\theta, X\rangle+2 \psi(\theta)) V_{n}(y+X / n)\right] \\
& =\inf _{\theta} E\left[\exp (-\langle\theta, X\rangle+\psi(\theta)) V_{n}(y+X / n)\right]
\end{aligned}
$$

for $y \notin B \cup C$ and subject to the boundary condition that $V_{n}(y)=1$ for $y \in B$ and $V_{n}(y)=0$ if $y \in C$. If there is an optimal policy $\left(\theta_{k}^{*}: k \geq 1\right)$ generated by the previous HJB equation, then generally it would be the case that

$$
\begin{aligned}
V_{n}(y) & =E \exp \left(-\sum_{k=1}^{n \tau_{B}(n)}\left\langle\theta_{k}^{*}, X_{k}\right\rangle+\sum_{k=1}^{n \tau_{B}(n)} \psi\left(\theta_{k}^{*}\right)\right) I\left(\tau_{B}(n)<\tau_{C}(n), \tau_{B}(n)<\infty\right) \\
& \leq E \exp \left(-\sum_{k=1}^{n \tau_{B}(n)}\left\langle\theta_{k}, X_{k}\right\rangle+\sum_{k=1}^{n \tau_{B}(n)} \psi\left(\theta_{k}\right)\right) I\left(\tau_{B}(n)<\tau_{C}(n), \tau_{B}(n)<\infty\right),
\end{aligned}
$$

for any non-anticipating policy $\left(\theta_{k}: k \geq 1\right)$.

The large deviations scaling suggests writing $V_{n}(y)=\exp \left(-n H_{n}(y)\right)$ and thus we should expect $H_{n}(y) \rightarrow H(y)$ as $n \rightarrow \infty$ for some function $H(y)$. If we proceed using this postulated limit in the previous HJB equation, after taking logarithms of (5), formally and without being careful about underlying smoothness assumptions and errors incurred, we arrive at the approximation

$$
\begin{aligned}
-n H(y) & \approx \min _{\theta} \log E[\exp (-\langle\theta, X\rangle+\psi(\theta)-n H(y+X / n))] \\
& \approx \min _{\theta} \log E[\exp (-\langle\theta, X\rangle+\psi(\theta)-n H(y)-\langle\nabla H(y), X\rangle)] .
\end{aligned}
$$

Equivalently, we have that

$$
\begin{aligned}
0 & \approx \min _{\theta} \log E[\exp (-\langle\theta, X\rangle+\psi(\theta)-\langle\nabla H(y), X\rangle)] \\
& =\min _{\theta} \log \exp (\psi(\theta)+\psi(-\nabla H(y)-\theta))=\min _{\theta}[\psi(\theta)+\psi(-\nabla H(y)-\theta)] .
\end{aligned}
$$

First order optimality conditions imply that at the optimal value $\theta^{*}(y)$ one has $\nabla \psi\left(\theta^{*}(y)\right)=$ $\nabla \psi\left(-\nabla H(y)-\theta^{*}(y)\right)$, which yields $\theta^{*}(y)=-\nabla H(y) / 2$ and therefore we conclude that equation (7) can be expressed as

$$
2 \psi(-\nabla H(y) / 2)=0,
$$




\section{Blanchet and Lam}

subject to the boundary conditions (inherited from (5)) equal to $H(y)=0$ for $y \in B$ and $H(y)=\infty$ for $y \in C$. Equation (8) corresponds to a so-called Isaacs equation. The name originates from a connection that can be established to a solution of a two-player differential game, see Dupuis and Wang (2004).

On the other hand, we have assumed $\alpha_{n}(y)=\exp \left(-n I_{B, C}(y)+o(n)\right)$ as $n \rightarrow \infty$ and, by conditioning on the first increment of the random walk, we also have that

$$
\alpha_{n}(y)=E \alpha_{n}(y+X / n)
$$

subject to the constraints that $\alpha_{n}(y)=1$ for $y \in B$ and $\alpha_{n}(y)=0$ if $y \in C$. Proceeding to analyze equality (9) formally as we did for the discussion leading to (8), we conclude that

$$
\exp \left(-n I_{B, C}(y)+o(n)\right)=E \exp \left(-n I_{B, C}(y+X / n)\right) \approx E \exp \left(-n I_{B, C}(y)-\left\langle\nabla I_{B, C}(y), X\right\rangle\right) .
$$

Equivalently, taking logarithms we arrive at $\psi\left(-\nabla I_{B, C}(y)\right)=0$, subject to the boundary conditions implied by $\alpha_{n}(y)$, namely, $I_{B, C}(y)=0$ for $y \in B$ and $I_{B, C}(y)=\infty$ if $y \in C$. These considerations, together with our analysis leading to (8) yield that $H(y)=2 I_{B, C}(y)$ and therefore, under suitable regularity conditions we obtain that applying importance sampling with exponential tilting given by the tilting parameter $\theta^{*}(y)=-\nabla I_{B, C}(y)$ allows us to obtain an asymptotically optimal estimator (recall Definition 2). Sufficient conditions required to rigorously substantiate this result are given in Dupuis and Wang (2004), at least in the case of large deviations problems for $S_{n} / n$.

The problem with (8) is that it is rarely applicable in most situations of interest, especially because it is often the case that there is no classical (smooth) solution to the Isaacs equation (8). (It is apparent from our heuristic derivation that smoothness appears to be an important feature.) The function $H(y)=2 I_{B, C}(y)$ typically provides a solution only in a weak sense. Fortunately, as we shall see next, only a subsolution is required, as long as it coincides with the value function at the initial condition.

\subsection{Subsolutions to a Deterministic Control Problem and Efficient Importance Sampling}

We take as starting point the HJB equation introduced in (5). The next lemma provides an inequality whose solution gives an upper bound for the value function in (5); for a proof see Blanchet and Lam (2011).

Lemma 1 Suppose that one finds a non-negative function $U_{n}(\cdot)$ such that

$$
U_{n}(y) \geq E\left[\exp (-\langle\theta, X\rangle+\psi(\theta)) U_{n}(y+X / n)\right] \geq \inf _{\theta} E\left[\exp (-\langle\theta, X\rangle+\psi(\theta)) U_{n}(y+X / n)\right],
$$

where $y \notin B \cup C$ and subject to the boundary condition that $U_{n}(y) \geq 1$ for $y \in B$. Then,

$$
U_{n}(y) \geq E\left[\exp \left(-\sum_{j=1}^{\tau_{B}}\left\langle\theta_{j}, X_{j}\right\rangle+\sum_{j=1}^{\tau_{B}} \psi\left(\theta_{j}\right)\right) I\left(\tau_{B}<\infty, \tau_{B}<\tau_{C}\right)\right]
$$

for any non-anticipating policy $\left(\theta_{j}: j \geq 1\right)$.

Using a similar heuristic argument as the one introduced in the previous section in (6) we write $U_{n}(y)=\exp \left(-n G_{n}(y)\right)$ and postulate $G_{n}(y) \rightarrow G(y)$ as $n \rightarrow \infty$ for some function $G(y)$. If we proceed using this postulated limit in the inequality obtained in the previous lemma, after taking logarithms, we arrive at

$$
\begin{aligned}
-n G(y) & \gtrsim \min _{\theta} \log E[\exp (-\langle\theta, X\rangle+\psi(\theta)-n G(y+X / n))] \\
& \approx \min _{\theta} \log E[\exp (-\langle\theta, X\rangle+\psi(\theta)-n G(y)-\langle\nabla G(y), X\rangle)] .
\end{aligned}
$$




\section{Blanchet and Lam}

We then conclude, as in the analysis leading to (7) that

$$
0 \geq \min _{\theta}[\psi(\theta)+\psi(-\nabla G(y)-\theta)]
$$

First order optimality conditions imply that at the optimal value $\theta^{*}(y)$ one has $\nabla \psi\left(\theta^{*}(y)\right)=$ $\nabla \psi\left(-\nabla G(y)-\theta^{*}(y)\right)$, which yields $\theta^{*}(y)=-\nabla G(y) / 2$ and therefore we conclude that inequality (10) can be expressed as

$$
0 \geq 2 \psi(-\nabla G(y) / 2)
$$

for $y \notin B \cup C$ and subject to the boundary conditions (inherited from Lemma 1) equal to $G(y) \leq 0$ for $y \in B$. In order to conclude asymptotic optimality for $\alpha_{n}\left(y_{0}\right)$ we must have $G\left(y_{0}\right) \geq 2 I_{B, C}\left(y_{0}\right)$. Sufficient conditions required to rigorously substantiate this subsolution equation are given in Theorem 8.1 of Dupuis and Wang (2007).

We now apply the previous result to an example, which is a generalization of a one dimensional ruin problem.

Example 1 (multidimensional first passage time problems) Assume that $d=2$ and let us write $X_{i}^{(j)}$ to denote the $j$-th component of the $i$-th increment; in particular, we have that $j=1,2$. Similarly, we use $Y_{n}^{(i)}(t)$ to denote the $i$-th component of the process $Y_{n}(\cdot)$ at time $t$, recall equation 2 . Assume that $X_{i}$ follows a bivariate Gaussian distribution with mean $E X_{i}=(-1,-1)^{T}$ and variance $\operatorname{Var}\left(X_{i}\right)=I$ (that is, the components of $X_{i}$ are independent). Therefore, we have that $\psi(\theta)=\|\theta\|^{2} / 2$.

Define the set $B(i)=\left\{y=\left(y^{(1)}, y^{(2)}\right)^{T}: y^{(i)} \geq 1\right\}$ and let $B=B(1) \cup B(2)$. Consider the first passage time probability

$$
\alpha_{n}(y)=P\left(\tau_{B}(n)<\infty \mid Y_{n}(0)=y\right),
$$

where $\tau_{B}(n)=\inf \left\{t \geq 0: Y_{n}(t) \in B\right\}$. We will construct an efficient estimator for $\alpha_{n}(y)$ based on (11).

Similarly, we let $\tau_{B(i)}(n)=\inf \left\{t>1: Y_{n}^{(i)}(t) \geq 1\right\}$ for each $i=1,2$, and note that one has the elementary inequalities

$$
\max _{i=1,2} P\left(\tau_{B(i)}(n)<\infty \mid Y_{n}(0)=y\right) \leq \alpha_{n}(y) \leq \sum_{i=1}^{2} P\left(\tau_{B(i)}(n)<\infty \mid Y_{n}(0)=y\right) .
$$

Evidently, $P\left(\tau_{B(i)}(n)<\infty \mid Y_{n}(0)=y\right)$ is equivalent to a one dimensional first passage time probability. It is well known (see Asmussen 2003 p. 352) that

$$
P\left(\tau_{B(i)}(n)<\infty \mid Y_{n}(0)=y\right)=\exp \left[-n \theta_{i}^{*}\left(1-y^{(i)}\right)+o(n)\right]
$$

where $\theta_{i}^{*}=2$. We then obtain from (12) that $\lim _{n \rightarrow \infty} n^{-1} \log \alpha_{n}(y)=-\min _{i=1,2}\left\{\theta_{i}^{*}\left(1-y^{(i)}\right)\right\}$. The value of $\theta_{i}^{*}$ corresponds to the conditional asymptotic mean of the $i-t h$ component if the random walk hits $B$ through $B(i)$.

To apply the subsolution approach we need to find a function $G(\cdot)$ satisfying the inequality (11) together with the corresponding boundary condition that $G(y) \leq 0$ for $y \in B$. Moreover, since in the end we are after an asymptotic upper bound for the second moment of the importance sampling estimator and we wish to achieve the same decay rate as $\alpha_{n}(y)^{2}$, it makes sense to use the union bound (12) and (13) to postulate

$$
\exp (-n G(y))=\left(\sum_{i=1}^{2} \exp \left[-n \theta_{i}^{*}\left(1-y^{(i)}\right)\right]\right)^{2} .
$$

We now verify that our selection is indeed a valid subsolution. First, note that $\nabla G(y)=-2 \sum_{i=1}^{2} \theta_{j}^{*} a_{j} p_{j}(y)$, where $a_{1}=(1,0), a_{2}=(0,1)$ and

$$
p_{j}(y, n)=\frac{\left.\exp \left[-n \theta_{j}^{*}\left(1-y^{(j)}\right)\right]\right)}{\sum_{i=1}^{2} \exp \left[-n \theta_{i}^{*}\left(1-y^{(i)}\right)\right]} .
$$




\section{Blanchet and Lam}

Note that for each $y,\left(p_{1}(y, n), p_{2}(y, n)\right)$ can be interpreted as a probability vector. So, using Jensen's inequality and convexity of $\psi(\cdot)$ we conclude that

$$
\psi(-\nabla G(y) / 2)=\psi\left(\sum_{j=1}^{2} \theta_{j}^{*} a_{j} p_{j}(y, n)\right) \leq \sum_{j=1}^{2} p_{j}(y, n) \psi\left(\theta_{j}^{*} a_{j}\right)=0 .
$$

In the last equality we used that $\psi\left(\theta_{j}^{*} a_{j}\right)=0$ for $j=1,2$. Now, if $y \in B$, then there exists $i$ such that $1-y^{(i)} \leq 0$ and therefore evidently $\exp (-n G(y)) \geq 0$ or, equivalently, $G(y) \leq 0$. We then conclude that the importance sampling scheme generated by using as exponential tilting

$$
\theta_{k}\left(S_{k-1} / n\right)=\sum_{j=1}^{2} \theta_{j}^{*} a_{j} p_{j}\left(S_{k-1} / n, n\right)
$$

for $k / n<\tau_{B}(n)$ is asymptotically optimal; actually in this case the estimator can be shown to be strongly efficient in the sense of Definition 1.

\section{STATE-DEPENDENT TECHNIQUES FOR HEAVY-TAILED SYSTEMS}

As mentioned earlier, various challenges of rare-event simulation with heavy tails are pointed out in Asmussen et al. (2000) and Bassamboo et al. (2006). The former focuses on the singularity of the asymptotic conditional distribution with respect to the original measure and the difficulty of finding a suitable importance sampler, while suggesting provably efficient conditional Monte Carlo scheme. The latter suggests the non-existence of provably efficient state-independent importance samplers for some first passage problems. Conditional Monte Carlo is also studied in Asmussen and Binswanger (1997) in the context of insurance, and further improved in Asmussen and Kroese (2006). A technique called hazard rate twisting is studied in Juneja et al. (1999) and Juneja and Shahabuddin (2002).

Our goal here is to illustrate a technique, based on Lyapunov inequalities and appropriate mixture samplers to estimate rare event probabilities. The Lyapunov approach was proposed in Blanchet and Glynn (2008) and it has subsequently applied to various settings (see, for instance, Blanchet et al. 2007; Blanchet and Liu 2008; Blanchet and Liu 2010b). The mixture distributions that we shall use were proposed in Dupuis et al. (2006) in the context of power-law decaying tails; see also Blanchet and Liu (2010a) for extensions beyond power-law decaying tails.

We first start with some basic results on heavy-tailed large deviations.

\subsection{Large Deviations for Heavy-tailed Sums}

For simplicity we concentrate on a special case of heavy-tailed random variables, namely, regularly varying random variables, which posses power-law type tails; see Embrechts et al. (1997) for more information on more general heavy-tailed models.

Definition 3 A random variable $X$ with tail distribution $\bar{F}(\cdot)=P(X>\cdot)=1-F(\cdot)$ has a regularly varying right tail with index $\alpha>0$ if $\bar{F}(\beta x) / \bar{F}(x) \longrightarrow \beta^{-\alpha}$ as $x \nearrow \infty$ for all $\beta>0$. Similarly, $X$ has a regularly varying left tail with index $\alpha>0$ if $-X$ has a regularly varying right tail with index $\alpha>0$.

A function $L(\cdot)$ is slowly varying at infinity if $L(\beta x) / L(x) \longrightarrow 1$ as $x \rightarrow \infty$ for each $\beta>0$. Therefore, if $X$ has regularly varying right tail with index $\alpha>0$ we often write $\bar{F}(t)=t^{-\alpha} L(t)$.

The following result provides insight on how large deviations tend to occur in heavy-tailed models.

Proposition 1 Suppose that $S_{m}=X_{1}+\ldots+X_{m}$ where the $X_{i}$ 's are iid non-negative regularly varying rv's. Then $P\left(\max _{1 \leq j \leq m} X_{j}>n \mid S_{m}>n\right) \longrightarrow 1$ as $n \nearrow \infty$.

The previous proposition illustrates the so-called "catastrophe principle" behind extreme behavior of heavy-tailed systems, which postulates that large deviations are caused by extremes in one or few components. In this case, the sum is large because one single component, namely the largest one, is large. 


\section{Blanchet and Lam}

In the heavy-tailed case there is a powerful heuristic that allows to "guess" a plausible form for large deviations results and the associated "most likely paths" to the rare event. This heuristic, which is known as the "fluid heuristic", is based on elementary Law of Large Numbers analysis and basic facts of regularly varying distributions. In order to guide our discussion we shall revisit a one dimensional analogue of the example studied earlier in the light tailed case.

Example 2 (first passage time probabilities for heavy-tailed random walk) Suppose that the $X_{j}$ 's are regularly varying with index $\alpha>1$. Assume that $E X_{k}=-\mu<0$ and let $S_{0}=y$. Define $\tau_{n}=\inf \left\{k \geq 0: S_{k}>n\right\}$ and consider the problem of approximating the ruin probability

$$
\alpha_{n}(y)=P\left(\tau_{n}<\infty \mid S_{0}=y\right)
$$

as $n \nearrow \infty$.

The fluid heuristic consists in substituting the random walk by its fluid or Law of Large Numbers behavior prior to the big jump that causes ruin. The associated fluid path in this case is $S_{\lfloor n t\rfloor} / n \approx y(t)=y-\mu t$ and therefore the fluid heuristic suggests

$$
\alpha_{n}(y) \sim \sum_{k=0}^{\infty} P\left(X_{k+1}+y(k)>n\right) \sim \int_{0}^{\infty} P(X>n+\mu t-y) d t
$$

as $n \nearrow \infty$, thereby neglecting the contribution of more than one jump and fluctuations beyond the Law of Large Numbers. Letting $u=n+\mu t-y$ we obtain

$$
\alpha_{n}(y) \sim \frac{1}{\mu} \int_{n-y}^{\infty} P(X>u) d y
$$

as $n \nearrow \infty$. This approximation turns out to be correct even beyond the assumption of regularly varying service times (see Pakes 1975; Veraverbeke 1977; Embrechts and Veraverbeke 1982).

\subsection{Conditional Distributions and Implications For Simulation}

As we discussed in Section 2, it is useful to understand the conditional distribution of the random walk given the occurrence of the rare event of interest because such conditional distribution provides the optimal changeof-measure (in terms of variance minimization). A natural starting point would be to obtain a conditional limit theorem for the $X_{k}$ 's given the rare event of interest. The asymptotic conditional distribution would then be a natural candidate for an importance sampling distribution. One can pursue this idea in the context of Example 2. In such case, it is not difficult to verify that for any $-\infty<x_{i}^{-}<x_{i}^{+}<\infty$, with $i=1, \ldots, k$ we have that

$$
\lim _{n \rightarrow \infty} P\left(\cap_{i=1}^{k}\left\{X_{i} \in\left(x_{i}^{-}, x_{i}^{+}\right)\right\} \mid \tau_{n}<\infty\right)=P\left(\cap_{i=1}^{k}\left\{X_{i} \in\left(x_{i}^{-}, x_{i}^{+}\right)\right\}\right) .
$$

Thus, concluding that the asymptotic conditional distribution of $\left(X_{1}, \ldots, X_{k}\right)$ given that $\tau_{n}<\infty$ remains unchanged as $n \rightarrow \infty$. This is one of the main reasons that makes the design of efficient importance sampling estimators for heavy-tailed systems a challenging problem.

Let us now take a more direct approach to approximate the increment $X_{k+1}$ given the current position $S_{k}=s$ of the random walk at time $k$ and given that $k<\tau_{n}<\infty$. We note (adopting the notation introduced in (3)) that

$$
\begin{aligned}
& P\left(X_{k+1} \in d y \mid \tau_{n} \in[k+1, \infty), S_{k}=s\right) \\
& =P\left(X_{k+1} \in d y, \tau_{n}=k+1 \mid \tau_{n} \in[k+1, \infty), S_{k}=s\right) \\
& +P\left(X_{k+1} \in d y, \tau_{n}>k+1 \mid \tau_{n} \in[k+1, \infty), S_{k}=s\right) .
\end{aligned}
$$




\section{Blanchet and Lam}

Now,

$$
P\left(X_{k+1} \in d y, \tau_{n}=k+1 \mid \tau_{n} \in(k, \infty), S_{k}=s\right)=p_{n}^{*}(s) \frac{P\left(X_{k+1} \in d y\right) I(y>n-s)}{P\left(X_{k+1}>n-s \mid s\right)},
$$

where $p_{n}^{*}(s)=P\left(X_{k+1}>n-s\right) / \alpha_{n}(s)$. Using the approximation in Example 2, we have that

$$
p_{n}^{*}(s) \sim p(n-s):=\mu \frac{P\left(X_{k+1}>n-s\right)}{\int_{n-s}^{\infty} P\left(X_{k+1}>t\right) d t} \sim \frac{\mu(\alpha-1)}{(n-s)}
$$

as $n-s \rightarrow \infty$. On the other hand, we have that

$$
\begin{aligned}
& P\left(X_{k+1} \in d y, \tau_{n}>k+1 \mid \tau_{n} \in[k+1, \infty), S_{k}=s\right) \\
& =\frac{P\left(X_{k+1} \in d y, \tau_{n} \in[k+2, \infty) \mid S_{k}=s\right) I(y \leq n-s)}{\alpha_{n}(s)} \\
& =\frac{P\left(X_{k+1} \in d y\right) I(y \leq n-s)}{P\left(X_{k+1} \leq n-s\right)} \times P\left(X_{k+1} \leq n-s\right) \frac{\alpha_{n}(s+y)}{\alpha_{n}(s)} .
\end{aligned}
$$

If we apply the fluid heuristic locally, that is by replacing $X_{k+1}$ by its mean, namely $E X_{k+1}=-\mu$, we arrive at

$$
\frac{\alpha_{n}\left(s+X_{k+1}\right)}{\alpha_{n}(s)} \approx \frac{\alpha_{n}(s-\mu)}{\alpha_{n}(s)} \approx \frac{(n-s+\mu)^{-\alpha+1}}{(n-s)^{-\alpha+1}} \approx[1+\mu /(n-s)]^{-\alpha+1}=1-\frac{\mu(1-\alpha)}{n-s}+o\left(\frac{1}{n-s}\right) .
$$

Therefore, applying the previous local version of the fluid heuristic into (16) and combining this with (15) we arrive at the non-rigorous approximation

$$
\begin{aligned}
P\left(X_{k+1} \in d y \mid \tau_{n} \in[k+1, \infty), S_{k}=s\right) & \approx \frac{P\left(X_{k+1} \in d y\right) I(y>n-s)}{P\left(X_{k+1} \leq n-s\right)} p(n-s) \\
& +\frac{P\left(X_{k+1} \in d y\right) I(y \leq n-s)}{P\left(X_{k+1} \leq n-s\right)}[1-p(n-s)] .
\end{aligned}
$$

It turns out that this approximation is indeed valid in total variation (Blanchet and Liu 2010a) as $n \rightarrow \infty$.

\subsection{State-dependent Importance Sampling Techniques for Heavy-tailed Random Walks}

We now discuss Lyapunov inequalities, which were introduced in Blanchet and Glynn (2008) in the context of importance sampling, to show the efficiency of the family of samplers suggested by (17). This technique is closely related and in some sense parallel to the subsolution approach explained earlier in the light-tailed setting.

Lemma 2 Suppose that there exists $r(x, y)>0$ satisfying

$$
\int r^{-1}(x, y) P\left(S_{j} \in d y \mid S_{j-1}=x\right)=1
$$

for all $x$ and $j \in\{1,2, \ldots\}$. Then, we can define a Markov transition kernel via $K(x, d y)=r^{-1}(x, y) P\left(S_{j} \in\right.$ $\left.d y \mid S_{j-1}=x\right)$ and an importance sampling estimator of the form

$$
R=\prod_{j=1}^{T_{A} \wedge T_{B}} r\left(S_{j-1}, S_{j}\right) I\left(T_{A}<T_{B}\right) .
$$

Suppose that there exists a non-negative function $G(\cdot)$ and a constant $\rho \in(0, \infty)$ such that

$$
E\left[r\left(x, S_{1}\right) G\left(S_{1}\right) \mid S_{0}=x\right] \leq G(x)
$$

for $x \notin A \cup B$ and $G(x) \geq \rho$ for $x \in A$. Then, $E\left[R \mid S_{0}=x\right] \leq G(x) / \rho$. 


\section{Blanchet and Lam}

The proof of the previous result is completely analogous to Lemma 1 (see Blanchet and Lam 2011). We now explain how to apply the previous result, combined with the fluid heuristics to design efficient importance sampling estimators in the context of Example 2.

Example 3 (Lyapunov inequalities and state-dependent importance sampling) We shall apply importance sampling following the analysis in Section 4.2. We distinguish two situations; first, if the current position $s$ is sufficiently far away from the boundary $n$, say if $n-s>\Delta$ for some $\Delta>0$, then the intuition developed in Section 4.2 is accurate and then we apply importance sampling using the mixture family.

However, in the second situation, if $s$ is sufficiently close to $n$ we do not need to apply importance sampling. Also, if we use the previous family when $s$ is sufficiently close to $n$, we might create variance because the mixture sampling distribution is derived using the large deviations regime, so turning off the sampling when $s$ is sufficiently close to $n$ is a sensible defense mechanism. We introduce a boundary layer $0 \leq n-s \leq \Delta$ for some $\Delta>0$ and if $s$ lies in this layer no importance sampling is applied.

Our family, then, takes the form

$$
\begin{aligned}
\widetilde{P}\left(X_{k+1}\right. & \left.\in d y \mid S_{k}=s\right)=\frac{P\left(X_{k+1} \in d y\right) I(y>a(n-s))}{P\left(X_{k+1}>a(n-s)\right)} p(n-s) I(n-s>\Delta) \\
& +\frac{P\left(X_{k+1} \in d y\right) I(y \leq a(n-s))}{P\left(X_{k+1} \leq a(n-s)\right)}[1-p(n-s)] I(n-s>\Delta) \\
& +P\left(X_{k+1} \in d y\right) I(n-s \leq \Delta) .
\end{aligned}
$$

The parameter $a \in(0,1)$ is selected in order to enforce uniform integrability conditions, as we shall see. Following the intuition developed earlier we propose

$$
p(n-s)=\theta P(X>a(n-s)) / \int_{n-s}^{\infty} P(X>t) d t,
$$

for some $\theta>0$. In fact, we expect $\theta \approx \mu$ to be optimal in the sense of variance minimization.

For notational convenience define $H(n-s)=\int_{n-s}^{\infty} P(X>t) d t$. We now need to construct a valid Lyapunov function. We wish to prove strong efficiency, so it is natural to suggest

$$
G(n-s)=\min \left(\kappa H^{2}(n-s), 1\right)=O\left(\alpha_{n}(s)^{2}\right)
$$

for some $\kappa>0$. The strategy is then to select (given $a \in(0,1)) \theta, \kappa$ and $\Delta$ in order to satisfy the Lyapunov inequality. If we can show that there exists a selection of these parameters that satisfies (18), then, given that the corresponding boundary condition in Lemma 2, namely

$$
G(n-s) \geq \rho:=\min \left(\kappa \int_{0}^{\infty} P(X>t) d t, 1\right)=\min \left(\kappa E X^{+}, 1\right)>0
$$

is satisfied for $s>n$, we would conclude strong efficiency of the estimator.

Note that the inequality (18) holds trivially if $G(n-s)=1$ is equivalent to $n-s \leq \Delta$. Indeed, in this case, since $G(x) \leq 1$ for every $x \in R$, inequality (18) takes the form $E G(n-s-X) \leq G(n-s)=1$.

Now observe that $G(n-s)<1$ holds if and only if $H(n-s)<1 / \kappa^{1 / 2}$, which in turn holds if and only if $n-s>H^{-1}\left(1 / \kappa^{1 / 2}\right)$. Therefore we can simply choose $\Delta=H^{-1}\left(1 / \kappa^{1 / 2}\right)$ and simply select $\theta$ and $\kappa$. In other words, we shall apply importance sampling using the mixture family if and only if our current position $s$ is such that $G(n-s)<1$. In order to proceed with the verification of inequality (18) we henceforth assume that $G(n-s)<1$. In this case inequality (18) takes the form

$$
J_{1}+J_{2} \leq 1
$$




\section{Blanchet and Lam}

where

$$
\begin{aligned}
& J_{1}=\frac{E[G(n-s-X) ; X>a(n-s)] P(X>a(n-s))}{\kappa H^{2}(n-s) p(n-s)}, \\
& J_{2}=\frac{E[G(n-s-X) ; X \leq a(n-s)] P(X \leq a(n-s))}{\kappa H^{2}(n-s)[1-p(n-s)]} .
\end{aligned}
$$

We first bound the term $J_{1}$. Observe, noting that $G(\cdot) \leq 1$, and using the form of $p(n-s)$, we obtain that

$$
J_{1} \leq \frac{P(X>a(n-s))}{\theta \kappa H(n-s)}=\frac{p(n-s)}{\theta^{2} \kappa} .
$$

Then we analyze the term $J_{2}$. Note that

$$
J_{2} \leq \frac{E\left[H^{2}(n-s-X) ; X \leq a(n-s)\right]}{H^{2}(n-s)[1-p(n-s)]} .
$$

Using the a Taylor expansion with remainder, expressed using elementary calculus with $t=x+y u$, we obtain

$$
H^{2}(x+y)-H^{2}(x)=\int_{x}^{x+y} 2 H(t) \dot{H}(t) d t=2 E[y H(x+y U) \dot{H}(x+y U)],
$$

where $U$ is uniformly distributed in $[0,1]$. Therefore, we have, letting $x=(n-s)$ and $y=-X$,

$$
\begin{aligned}
& \frac{E\left[H^{2}(n-s-X) I(X \leq a(n-s))\right]}{H^{2}(n-s)} \\
& =P(X \leq a(n-s))-2 \frac{E[X \dot{H}(n-s-X U) H(n-s-X U) ; X \leq a(n-s)]}{H^{2}(n-s)},
\end{aligned}
$$

where $X$ and $U$ are independent. Now,

$$
\begin{aligned}
& -\frac{E[X \dot{H}(n-s-X U) H(n-s-X U) ; X \leq a(n-s)]}{H^{2}(n-s)} \\
& =\frac{E[X \bar{F}(n-s-X U) H(n-s-X U) ; X \leq a(n-s)]}{H^{2}(n-s)} .
\end{aligned}
$$

We are assuming that $G(n-s)<1$, or equivalently, that $n-s>H^{-1}\left(1 / \kappa^{1 / 2}\right)=\Delta$. On the other hand, we have that

$$
X \frac{\bar{F}(n-s-X U) H(n-s-X U) I(X \leq a(n-s))}{H^{2}(n-s)} \sim X \frac{\alpha-1}{(n-s)}
$$

almost surely as $n-s \nearrow \infty$ and also we have, because of regular variation and Karamata's theorem (see for example Resnick 2004 p. 17) that there exists a constant $K \in(0, \infty)$ such that

$$
\begin{aligned}
& |X| \frac{\bar{F}(n-s-X U) H(n-s-X U) I(X \leq a(n-s))}{H^{2}(n-s)} \\
& \leq|X| \frac{\bar{F}((n-s)(1-a)) H((n-s)(1-a))}{H^{2}(n-s)} \leq \frac{K|X|}{n-s} .
\end{aligned}
$$


Consequently, if $\kappa$ (or equivalently $\Delta=H^{-1}\left(1 / \kappa^{1 / 2}\right)$ ) is chosen sufficiently large, we conclude, applying the dominated convergence theorem (note here that $a \in(0,1)$ has been crucial) that for each $\varepsilon>0$

$$
J_{2} \leq 1+2 E X \frac{(\alpha-1)}{n-s}(1-\varepsilon)
$$

as long as $G(n-s)<1$. Combining our estimates for $J_{1}$ and $J_{2}$ and choosing $\kappa$ sufficiently large so that

$$
p(n-s) \leq \theta a^{-\alpha} \frac{(\alpha-1)(1+\varepsilon)}{n-s}, \frac{1}{1-p(n-s)} \geq 1+\theta a^{-\alpha} \frac{(\alpha-1)(1-\varepsilon)}{n-s},
$$

we arrive at

$$
\begin{aligned}
J_{1}+J_{2} & \leq \frac{(\alpha-1)(1+\varepsilon)}{\theta \kappa(n-s)} a^{-\alpha}+\left(1-2 \mu \frac{(\alpha-1)}{n-s}(1-\varepsilon)\right)\left(1+\theta a^{-\alpha} \frac{(\alpha-1)(1-\varepsilon)}{n-s}\right) \\
& \leq a^{-\alpha} \frac{(\alpha-1)(1+\varepsilon)}{\theta \kappa(n-s)}+\left(1-2 \mu \frac{(\alpha-1)}{n-s}(1-\varepsilon)+\theta a^{-\alpha} \frac{(\alpha-1)(1-\varepsilon)}{n-s}\right) .
\end{aligned}
$$

In order to enforce (19) we then need to ensure that

$$
a^{-\alpha} \frac{1+\varepsilon}{\theta \kappa}-2 \mu(1-\varepsilon)+a^{-\alpha} \theta(1-\varepsilon)<0,
$$

which clearly feasible given that $\mu>0$. Note that it is crucial that $-\mu=E X<0$, otherwise it would be impossible to select the parameters appropriately. This makes sense given that when $E X \geq 0, \alpha_{n}(y)=1$

and it would be therefore impossible to bound the second moment of the estimator by a function that decreases to zero as $n \rightarrow \infty$, as we are proposing. See Blanchet and Liu (2010a) for further discussion on the associated running time of the sampler.

\section{ACKNOWLEDGEMENT}

Support from the NSF foundation through the grants DMS-0806145, DMS-0846816 and DMS-1069064 is gratefully acknowledged.

\section{REFERENCES}

Asmussen, S. 1985. "Conjugate processes and the simulation of ruin problems". Stochastic Processes and their Applications 20:213-229.

Asmussen, S. 2000. Ruin Probabilities. London, UK: World Scientific.

Asmussen, S. 2003. Applied Probability and Queues. New York, NY, USA: Springer-Verlag.

Asmussen, S., and K. Binswanger. 1997. "Simulation of Ruin probabilities for Subexponential Claims". ASTIN Bulletin 27:297-318.

Asmussen, S., K. Binswanger, and B. Hojgaard. 2000. "Rare events simulation for heavy-tailed distributions". Bernoulli 6:303-322.

Asmussen, S., and P. Glynn. 2007. Stochastic Simulation: Algorithms and Analysis. New York, NY, USA: Springer-Verlag.

Asmussen, S., and D. Kroese. 2006. "Improved algorithms for rare event simulation with heavy tails". Advances in Applied Probability 38:545-558.

Asmussen, S., and R. Rubinstein. 1995. "Steady state rare events simulation in queueing models and its complexity properties". In Advances in Queueing, edited by J. Dshalalow, 429-461. CRC Press.

Bassamboo, A., S. Juneja, and A. Zeevi. 2006. "On the inefficiency of state-independent importance sampling in the presence of heavy-tails”. Operations Research Letters 34:521-531. 


\section{Blanchet and Lam}

Blanchet, J., and P. Glynn. 2008. "Efficient rare-event simulation for the maximum of a heavy-tailed random walk.". Ann. of Appl. Probab. 18:1351-1378.

Blanchet, J., P. Glynn, and J. C. Liu. 2007. "Fluid heuristics, Lyapunov bounds and efficient importance sampling for a heavy-tailed $G / G / 1$ queue". QUESTA 57:99-113.

Blanchet, J., and H. Lam. 2011. "State-dependent importance sampling for rare-event simulation: An overview and recent advances". under review in Surveys in Operations Research and Management Sciences.

Blanchet, J., and J. C. Liu. 2008. "State-dependent importance sampling for regularly varying random walks". Adv. in Appl. Probab. 40:1104-1128.

Blanchet, J., and J. C. Liu. 2010a. "Efficient simulation and conditional functional limit theorems for ruinous heavy-tailed random walks". submitted, http://www.columbia.edu/ jb2814/papers/ConcaveHazard9f.pdf.

Blanchet, J., and J. C. Liu. 2010b. "Importance sampling in ruin problems for multidimensional regularly varying random walks". Journal of Applied Probability 47:301-322.

Bucklew, J. 1990. Large Deviation Techniques in Decision, Simulation and Estimation. New York, NY, USA: Wiley.

Chan, H. P., and T. L. Lai. 2010. "A sequential Monte Carlo approach to computing tail probabilities in stochastic models". Ann. of Appl. Prob., forthcoming.

Collamore, J. 2002. "Importance sampling techniques for the multidimensional ruin problem for general Markov additive sequences of random vectors". Ann. Appl. Probab. 12:382-421.

Dembo, A., and O. Zeitouni. 1998. Large deviations techniques and applications, 2 nd edition. New York, NY, USA: Springer.

Dupuis, P., K. Leder, and H. Wang. 2006. "Importance sampling for sums of random variables with regularly varying tails.”. ACM TOMACS 17.

Dupuis, P., K. Leder, and H. Wang. 2007. "Large deviations and importance sampling for a tandem network with slowdown.”. QUESTA 57:71-83.

Dupuis, P., A. Sezer, and H. Wang. 2007. "Dynamic importance sampling for queueing networks.”. Ann. Appl. Probab. 17:1306-1346.

Dupuis, P., and H. Wang. 2004. "Importance sampling, large deviations, and differential games". Stoch. Stoch. Reports 76:481-508.

Dupuis, P., and H. Wang. 2007. "Subsolutions of an Isaacs equation and efficient schemes of importance sampling". Mathematics of Operations Research 32:723-757.

Embrechts, P., C. Klppelberg, and T. Mikosch. 1997. Modelling Extremal Events for Insurance and Finance. New York: Springer-Verlag.

Embrechts, P., and N. Veraverbeke. 1982. "Estimates for the probability of ruin with special emphasis on the possibility of large claims". Insurance: Math. Economics 1:55-72.

Glasserman, P., and S. Kou. 1995. "Analysis of an importance sampling estimator for tandem queues". ACM Trans. Mod. Comp. Sim. 5:22-42.

Glasserman, P., and Y. Wang. 1997. "Counter examples in importance sampling for large deviations probabilities". Annals of Applied Probability 7:731-746.

Juneja, S., and P. Shahabuddin. 2002. "Simulating heavy tailed processes using delayed hazard rate twisting". ACM Trans. Model. Comput. Simul. 12 (2): 94-118.

Juneja, S., and P. Shahabuddin. 2006. "Rare event simulation techniques: An introduction and recent advances". Handbook on Simulation:291-350.

Juneja, S., P. Shahabuddin, and A. Chandra. 1999, December. "Simulating heavy tailed processes using delayed hazard rate twisting". In Proceedings of the 1999 Winter Simulation Conference, edited by P. A. Farrington, H. B. Nembhard, D. T. Sturrock, and G. Evans, 420-427. Piscataway, New Jersey: Institute of Electrical and Electronics Engineers, Inc. 


\section{Blanchet and Lam}

Kollman, C., K. Baggerly, D. Cox, and R. Picard. 1999. "Adaptive importance sampling on discrete Markov chains". Annals of Applied Probability 9:391-412.

L'Ecuyer, P., J. Blanchet, B. Tuffin, and P. Glynn. 2010. "Asymptotic robustness of estimators in rare-event simulation". ACM TOMACS 20:6:1-6:41.

L'Ecuyer, P., F. L. Gland, P. Lezaud, and B. Tuffin. 2009. "Splitting techniques". In Rare Event Simulation Using Monte Carlo Methods, edited by G. Rubino and B. Tuffin, 39-62. West Sussex, United Kingdom: Wiley. Chapter 3.

Pakes, A. G. 1975. "On the tails of waiting-time distribution". J. Appl. Probab. 12:555-564.

Parekh, S., and J. Walrand. 1989. "Quick simulation of rare events in networks". IEEE Trans. Automat. Contr. 34:54-66.

Resnick, S. I. 2004. Extreme Values, Regular Variation, and Point Processes. New York, USA: Springer.

Rubinstein, R., and D. Kroese. 2004. The Cross-Entropy Method. New York, NY: Springer.

Rubinstein, R. Y., and D. P. Kroese. 2009. Simulation and the Monte Carlo Method, 2nd Edition. John Wiley.

Sadowsky, J. S. 1991. "Large deviations theory and efficient simulation of excessive backlogs in a $G I / G I / m$ queue". IEEE Trans. Autom. Control 36:1383-1394.

Siegmund, D. 1976. "Importance sampling in the Monte Carlo study of sequential tests". Ann. Stat. 4:673684.

Veraverbeke, N. 1977. "Asymptotic behavior of Wiener-Hopf factors of a random walk". Stochastic Process. Appl. 5:27-31.

\section{AUTHOR BIOGRAPHIES}

JOSE BLANCHET is a faculty member of the IEOR Department at Columbia University. Jose holds a $\mathrm{Ph} . \mathrm{D}$. in Management Science and Engineering from Stanford University. Prior to joining Columbia he was a faculty member in the Statistics Department at Harvard University. Jose is a recipient of the 2009 Best Publication Award given by the INFORMS Applied Probability Society and of the 2010 Erlang Prize. He also received a PECASE award sponsored by NSF in 2010. He worked as an analyst in Protego Financial Advisors, a leading investment bank in Mexico. He has research interests in applied probability and Monte Carlo methods. He serves in the editorial board of Advances in Applied Probability, Journal of Applied Probability, Mathematics of Operations Research and QUESTA.

HENRY LAM is an Assistant Professor in the Department of Mathematics and Statistics at Boston University. He recently graduated from Harvard University with a Ph.D. degree in statistics. His research interests lie in applied probability and Monte Carlo methods with applications in queueing, operations management and insurance modeling. 